\title{
Simulation of Aqueous Humour Flow Driven by Buoyancy Effects and Flow Through Pupil Aperture During Descemet Membrane Detachment
}

\author{
Woon Woan Jen ${ }^{1}$, Zuhaila Ismail ${ }^{1,}$, Alistair Fitt ${ }^{2}$ \\ Department of Mathematical Science, Faculty of Science, Universiti Teknologi Malaysia, 81310 Skudai, Johor, Malaysia \\ Faculty of Technology, Design and Environment, Oxford Brookes University Headington Campus, Gipsy Lane, Oxford, OX3 OBP, United Kingdom
}

\section{ARTICLE INFO}

\section{Article history:}

Received 19 February 2020

Received in revised form 16 April 2020

Accepted 21 April 2020

Available online 28 April 2020

\section{ABSTRACT}

Descemet membrane detachment (DMD) is a tear or break on the Descement membrane (DM) which is a part of the cornea membrane. DMD is occurred when DM separate from stroma. The separation is due to the aqueous humour $(A H)$ flows into the subspaces between the stroma and DM. A mathematical model of buoyancy driven $\mathrm{AH}$ flow is developed to analyse the behaviour of the fluid flows through the pupil aperture in anterior chamber (AC) during DMD. Finite element method using COMSOL Multiphysics 5.2 is implemented to simulate the buoyancy driven flow of AH enters the AC across pupil aperture and leaves through Trabecular meshwork (TM) during DMD. Results are obtained for the eye in standing and supine position. The effects considered is the temperature differences between the cornea and iris that act as the driver of $\mathrm{AH}$ flow, variation of pupil size indicate the different level of ambient light and the influence of gravity on the deformation of DMD. Buoyancy effect is observed to be the dominant driven mechanism of $\mathrm{AH}$ flow in AC. Variation of pupil size has no effect on the pattern of the fluid flow in AC. The standing and supine position of human are able to change the recirculation pattern of $\mathrm{AH}$ in the $\mathrm{AC}$. Therefore, the reattachment of DMD is significantly affected by the body position of the patient.

Keywords:

Aqueous humour; descemet membrane detachment; buoyancy effect
Copyright $\odot 2020$ PENERBIT AKADEMIA BARU - All rights reserved

\section{Introduction}

Descement membrane detachment (DMD) is a complication that will threaten humans' sight after intraocular surgery. DMD is the tear or breaks on the Descement membrane (DM) which is located between the stroma and endothelium. In the cornea, there is an outermost and innermost layer called epithelium and endothelium respectively. Stroma is the intermediate layer. Anterior chamber that is filled by aqueous humour $(\mathrm{AH})$ is bounded by cornea, iris and pupil. $\mathrm{AH}$ is a transparent fluid which has similar properties to water. Once the DMD happens, the AH will flows into the subspaces between the stroma and DM and then separate the DM away from stroma.

\footnotetext{
* Corresponding author.

E-mail address: zuhaila@utm.my (Zuhaila Ismail)
} 
There are a lot of treatment option have been done to repair the case of DMD such as the injection of sulphur hexafluoride gas or viscoelastic as suggested in Sevillano et al., [1]. Potter et al., [2] suggested surgical repair, anterior chamber injection, and corneal transplantation. However, Ünlü et al., [3] reported that the exact mechanism of DMD still remains unknown. Couch et al., [4] discovered a case that a patient had DMD in both eye after surgery but both eye were recovered. One eye was treated surgically but the other eye improved spontaneously. Hence, they claimed that the spontaneous reattachment might be caused by the buoyancy-driven $\mathrm{AH}$ flow. It is believed that the condition of DMD can be controlled or improved by altering the gradient of temperature across the eye [5]. The verification of convection occurs due to temperature gradient induced by AH flow is conducted by Heys et al., [6]. The thermally driven flow is able to produce grater velocities than those others mechanism such as aqueous humor secretion, buoyancy-driven flow during sleep and interaction between buoyancy and gravity [7].

Many of the researchers have studied the impact of AH flow when there is DMD. Ismail et al., [8] analysed the interaction between the $\mathrm{AH}$ flow and deformation of DMD in three dimensional AC. Ooi et al., [9] simulated the two dimensional model of $A C$ and investigate the temperature distribution on the AH flow. They also investigated the effects of eye orientation on the $\mathrm{AH}$ flow and the temperature distribution. Besides, Loke et al., [10] discussed the effect of segmental outflow in AC on the drug delivery through AH flow.

Motivated by these previous studies, the objective of present study is to investigate the behaviour of AH flow through the pupil aperture in the presence of DMD by numerical simulation with the aid of COMSOL Multiphysics simulation software. The flow in porous region is modelled to represent trabecular meshwork (TM) that is responsible for the outflow of AH. An appropriate mathematical model by taking into account the characteristic of $\mathrm{AH}$ is developed to examine the behaviour of AH flow in AC. Computer simulation is used as the reproduction of the behaviour of fluid flow based on the mathematical model. COMSOL Multiphysics is able to simulate the outcomes of the mathematical model by produces realistic results with sketching and helps to enhance the analysis of fluid flow model [11]. Throughout this research can helps to explore the results from the analysis and get a deeper view of the phenomena of DMD which has a close relation with the AH flow. Hence, the treatment of DMD can be improved. The probability of DMD happen after surgery can be reduce with the knowledge of the behaviour of $\mathrm{AH}$ flow through pupil aperture.

\section{Methodology}

\subsection{Model Construction}

As shown in Figure 1, a two dimensional model of AC in human eye is developed in present study consist of cornea, iris, pupil, trabecular meshwork (TM), Schelemm's canal (SC) and DMD. The iris, lens, cornea and DMD in the AC are modelled as a rigid surface. The $\mathrm{AH}$ has the properties similar to water and it is secreted from ciliary body at the rate of $2.4 \mu \mathrm{l} / \mathrm{min}$ [12]. The AH enters the AC through the small gap between the iris and the lens and flow out through the TM to SC.

The buoyancy effect appears in $A C$ is due to the temperature gradient between the cornea and back of AC. The temperature at the back of AC is assumed the same as the temperature at iris or pupil and it is denoted as $T_{p}$. $T_{p}$ is estimated near to body temperature that is $37^{\circ} \mathrm{C}$ or $310.15 \mathrm{~K}$. While the cornea is exposed to ambient temperature which is estimated as $24^{\circ} \mathrm{C}$ hence the cornea temperature is slightly lower than temperature at iris. The temperature of cornea denoted as $T_{c}$ is estimated as $35^{\circ} \mathrm{C}$ or $308.15 \mathrm{~K}$. Since the cornea is considered as stationary tissues and thus the DMD which detached from part of the cornea is rigid. In reality, the DMD does not has a fixed shape. For simplicity, DMD is assumed to be a flat plate as shown in Figure 1. 


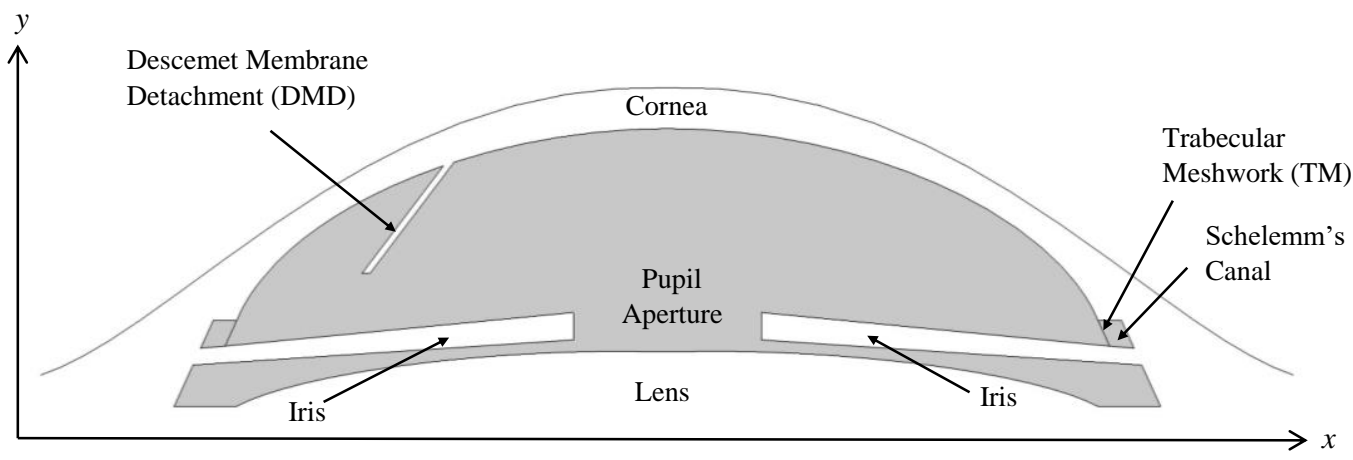

Fig. 1. Schematic diagram of Anterior Chamber of human eye with DMD

The $\mathrm{AH}$ is assumed to be incompressible, Newtonian and driven by buoyancy force. The natural convection occurs due to the temperature difference brings to the changing of the density of the fluid. Boussinesq approximation is used for the flow that is driven by temperature difference and assume that the fluid density varies slightly with temperature, but negligibly with pressure. Boussinesq approximation is applied in order to facilitate the procedure of solving the problem. Hence, the governing equations for the $\mathrm{AH}$ flow driven by temperature difference in $\mathrm{AC}$ are [13]

$\frac{\partial u}{\partial x}+\frac{\partial v}{\partial y}=0$

$-\frac{1}{\rho_{0}} \frac{\partial p}{\partial x}+v\left(\frac{\partial^{2} u}{\partial x^{2}}+\frac{\partial^{2} u}{\partial y^{2}}\right)-\left(u \frac{\partial u}{\partial x}+v \frac{\partial u}{\partial y}\right)+g\left(1-\beta\left(T-T_{c}\right)\right)=0$

$-\frac{1}{\rho_{0}} \frac{\partial p}{\partial y}+v\left(\frac{\partial^{2} v}{\partial x^{2}}+\frac{\partial^{2} v}{\partial y^{2}}\right)-\left(u \frac{\partial v}{\partial x}+v \frac{\partial v}{\partial y}\right)=0$

$\frac{k}{\rho_{0} c_{p}}\left(\frac{\partial^{2} T}{\partial x^{2}}+\frac{\partial^{2} T}{\partial y^{2}}\right)-\left(u \frac{\partial T}{\partial x}+v \frac{\partial T}{\partial y}\right)=0$

Here $\rho_{0}$ is the fluid density at temperature $T_{c}$ which is temperature at cornea, $\beta$ is coefficient of thermal expansion, $k$ is thermal conductivity of $A H$ and $c_{p}$ is the specific heat of $A H$. According to Ismail [5] and Canning et al., [14], a set of typical values of the properties of AH required for modelling the problem and values of parameter involved are shown in Table 1.

Table 1

Values of $\mathrm{AH}$ and parameters used in present study $[5,14]$

\begin{tabular}{lll}
\hline Parameter & Description & Unit \\
\hline$\beta$ & Coefficient of thermal expansion & $3 \times 10^{-4} \mathrm{~K}^{-1}$ \\
$\mathrm{c}_{p}$ & Specific heat of $\mathrm{AH}$ & $4200 \mathrm{Jkg}^{-1} \mathrm{~K}^{-1}$ \\
$\mathrm{~g}$ & Gravity & $9.8 \mathrm{~ms}^{-1}$ \\
$\mathrm{k}$ & Thermal conductivity of $\mathrm{AH}$ & $0.57 \mathrm{Wm}^{-1} \mathrm{~K}^{-1}$ \\
$\mu$ & Viscosity of $\mathrm{AH}$ & $0.9 \times 10^{-3} \mathrm{kgm}^{-3}$ \\
$\mathrm{U}$ & Kinematic viscosity of $\mathrm{AH}$ & $0.9 \times 10^{-6} \mathrm{~m}^{2} \mathrm{~s}^{-1}$ \\
$\rho$ & Density of $\mathrm{AH}$ & $1000 \mathrm{kgm}^{-3}$ \\
$\mathrm{~T}_{\mathrm{p}}$ & Temperature at iris/pupil & $310.15 \mathrm{~K}$ \\
$\mathrm{~T}_{\mathrm{c}}$ & Temperature at cornea & $308.15 \mathrm{~K}$ \\
\hline
\end{tabular}

The flow in porous region is modelled to representing the TM and can be described by StokesBrinkman equation [10]. The pressure gradient in the governing equations of $\mathrm{AH}$ flow in AC for Eq. 
(2) and Eq. (3) is used to describe the porosity of TM. According to Kumar et al., [15], the relative pressure gradient is

$\nabla p=-\frac{\mu}{\kappa} u+\frac{\mu}{\varepsilon} \nabla^{2} u$

where $\mathrm{K}$ and $\varepsilon$ is the permeability and porosity of the TM respectively. $\mu$ is the fluid viscosity. The value of permeability, $\mathrm{k}$ is taken as $2 \times 10^{-15} \mathrm{~m}^{2}$ and porosity $\varepsilon$ is 0.15 [10].

\subsection{Boundary Conditions}

Since the lens, iris and cornea are set as stationary surfaces, hence no slip condition is applied along all of this surface except the inlet and outlet boundaries. A flat inlet velocity representing the velocity magnitude of $\mathrm{AH}$ enter the $\mathrm{AC}$ through pupil aperture and it is assumed as $2 \times 10^{-6} \mathrm{~m} / \mathrm{s}$ which is related to the production rate of $\mathrm{AH}$ in posterior chamber [8]. Under normal condition, the pressure in aqueous vein is $9 \mathrm{mmHg}(1200 \mathrm{~Pa})$. Therefore, the pressure of $1200 \mathrm{~Pa}$ is used as the outlet boundary condition when the AH flow out through the porous medium TM from AC. The upper and lower surfaces of TM also applied the no slip boundary condition. The temperature of the surfaces where the $\mathrm{AH}$ flow in through the pupil aperture is denote as $\mathrm{T}_{\mathrm{p}}$ and it is assumed as $310.15 \mathrm{~K}\left(37^{\circ} \mathrm{C}\right)$. Since the upper part of AC which is cornea is exposed to ambient temperature so the temperature of cornea is slightly lower than $T_{p}$. The temperature drop is assumed to be $2^{\circ} \mathrm{C}$ therefore temperature of cornea denoted as $\mathrm{T}_{\mathrm{c}}$ is $308.15 \mathrm{~K}\left(35^{\circ} \mathrm{C}\right)$.

\subsection{Computational Mesh}

Throughout this study, finite element method is applied to compute the model by using COMSOL Multiphysics 5.2. The two dimensional model is meshed with triangular elements by applied physicscontrolled mesh setting sequence in COMSOL. Four different mesh sizes of normal, fine, finer and extra fine are tested and the maximum velocity generated for the four meshes are nearly the same. The mesh test results are shown in Table 2.Thus, the result did not depend on the number of the element used to mesh the geometry [12]. Finer element size is selected to solve the problem in this study.

Table 2

Mesh test for four different mesh size

\begin{tabular}{lllll}
\hline Mesh Size & Normal & Fine & Finer & Extra Fine \\
\hline Domain Element & 4730 & 7112 & 17688 & 45638 \\
Maximum Velocity $(\mathrm{m} / \mathrm{s})$ & $2.4230 \mathrm{E}-04$ & $2.4230 \mathrm{E}-04$ & $2.4277 \mathrm{E}-04$ & $2.4370 \mathrm{E}-04$ \\
\hline
\end{tabular}

\subsection{Model Validation}

The two dimensional thermal driven flow model is validated by comparing with the streamline plot in Canning et al., [14] and analytical solutions in Ismail et al., [8]. As shown in Figure 2, the direction of the flow in AC simulated in COMSOL Multiphysics is concurred with the streamline plot in Canning et al., [14] 


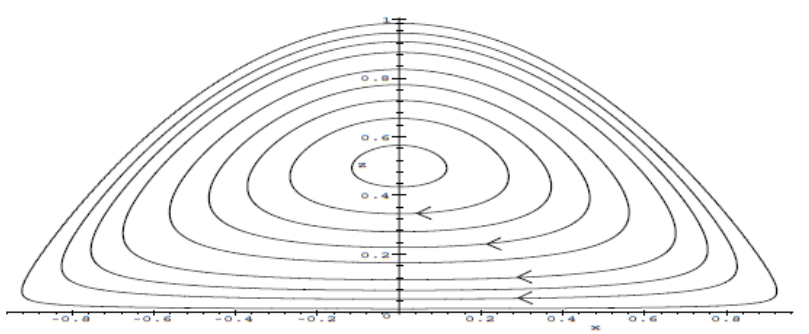

(a)

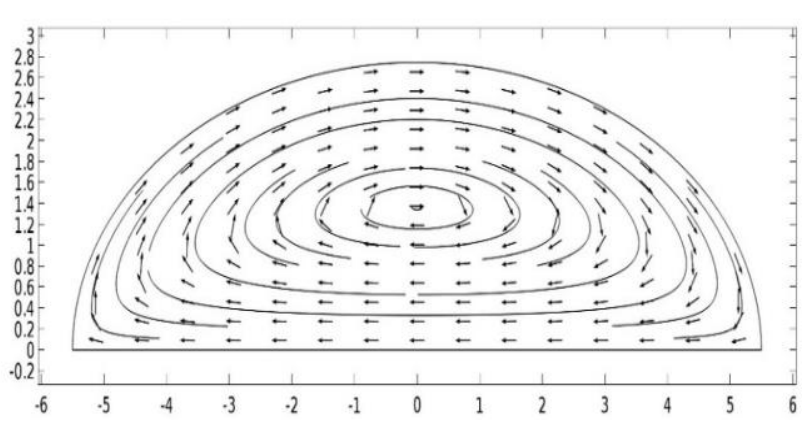

(b)

Fig. 2. Streamline plot of buoyancy driven flow (a) Streamline plot in Ethier et al., [12] (b) Streamline plot by COMSOL Multiphysics 5.2

Besides that, the maximum velocity computed in Figure $2(\mathrm{~b})$ is $3.473 \times 10^{-4} \mathrm{~m} / \mathrm{s}$. In Ismail et al., [8], the authors determined the maximum flow speed analytically which is $3.962 \times 10^{-4} \mathrm{~m} / \mathrm{s}$ for the same model. The difference between the maximum velocity magnitudes obtained by different calculations method is considered small and acceptable. Thus, this illustrated the good agreement of the current validation of the model.

After that, the model of $\mathrm{AH}$ flows through pupil aperture is validated with the streamline plot for flow through pupil aperture in Canning et al., [14] (Figure 3(a)). The streamline and velocity direction plot that simulated in COMSOL is coincided with Figure 3(a).

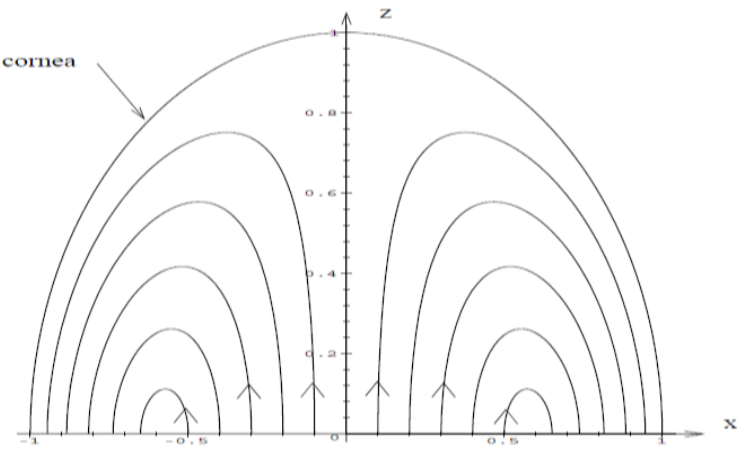

(a)

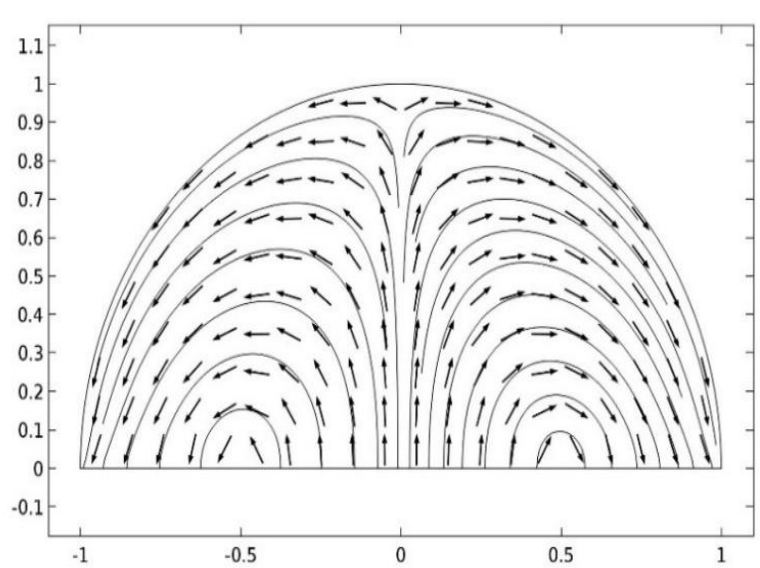

(b)

Fig. 3. Streamline plot of the flow through pupil aperture in AC (a) Streamline plot in Woan Jen [12]

(b) Streamline plot by COMSOL Multiphysics 5.2

\section{Result}

\subsection{Eye in Standing Position}

For an individual in standing position, the body and gravitational forces act in positive $x$-direction. Let's consider the behaviour of AH flow without DMD. The results showed that the fluid flow in AC creates a large symmetric recirculation zone as illustrated in Figure 4. The fluid enters the pupil and then move in negative $x$-direction along the iris surface. By encounters with higher resistance of the right TM region, the fluid then turns and moves along the corneal surfaces in positive $x$-direction towards the left side of the TM zone. By encounters with higher resistance of the left TM region, the 
fluid then turns and moves along the corneal surfaces in positive $x$-direction towards the right side of the TM zone. The highest velocity is $2.4375 \times 10^{-4} \mathrm{~m} / \mathrm{s}$ located at the centre of pupil aperture. Figure 4(c) displayed the pressure contour and the TM region at both sides have a significant effect due to the porosity properties of TM. The temperature contour is shown in Figure 4(d). The AH near the lens and iris has the warmest temperature while the coldest area existed along the corneal surface. Generally, fluid flows from warmer area to colder area. Hence, the behaviour of the AH flow is mainly depend on the temperature of the eye and driven by the temperature difference.

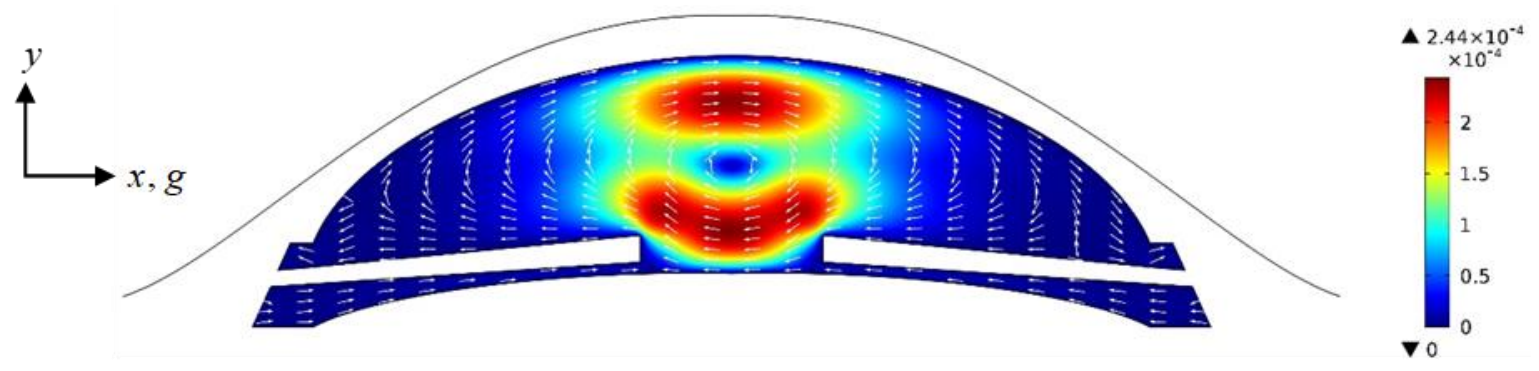

(a)

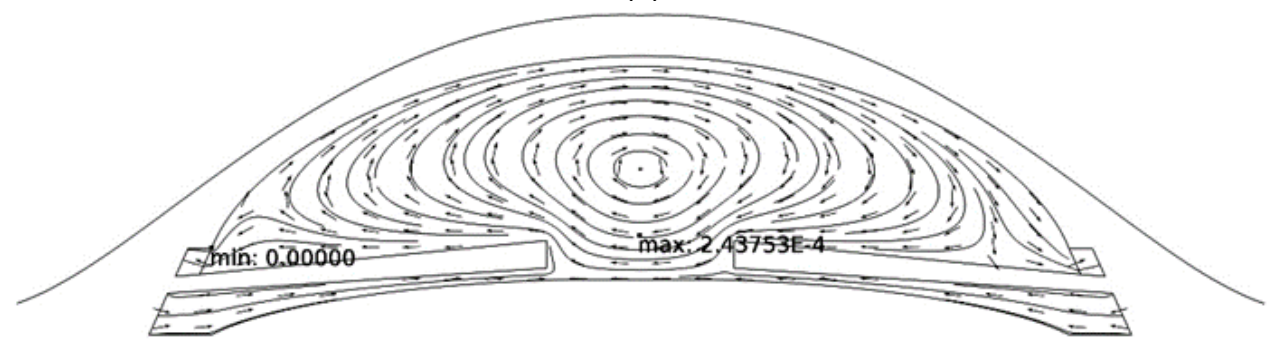

(b)

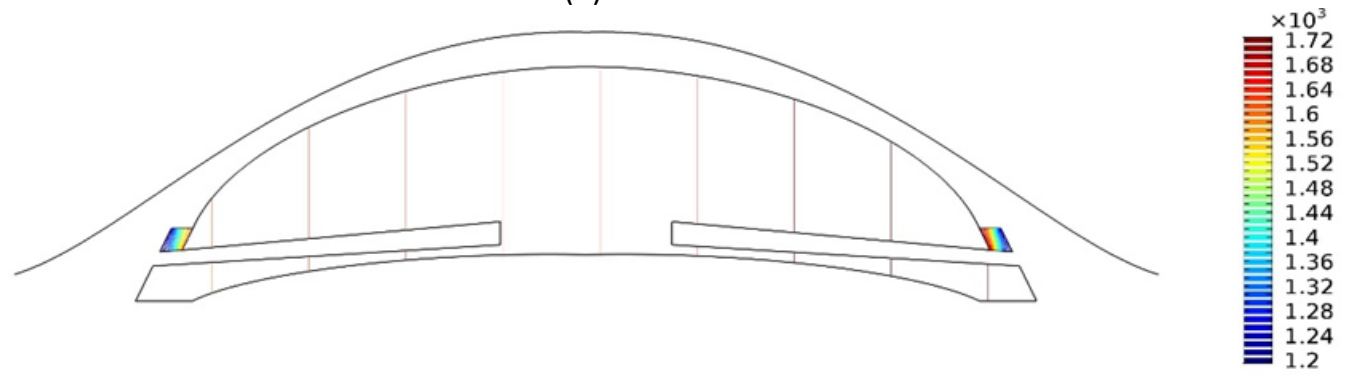

(c)

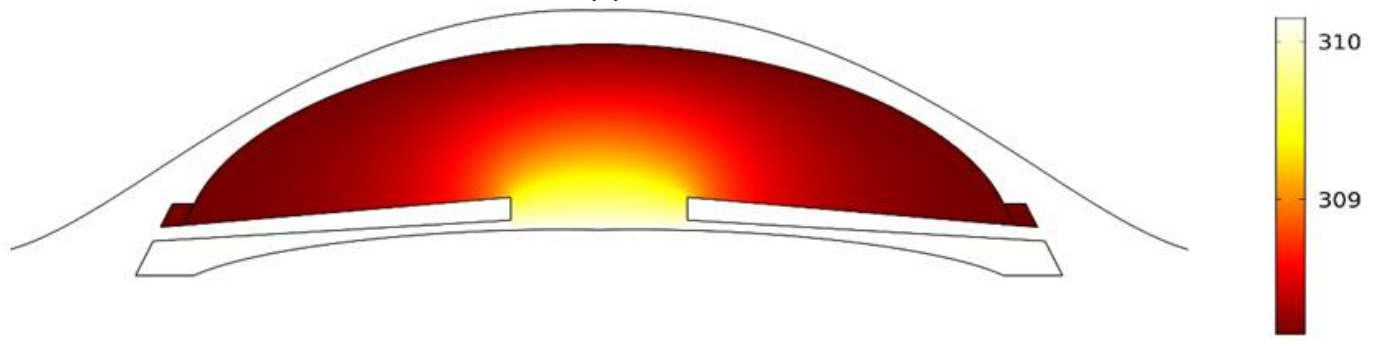

(d)

Fig. 4. AH flow for eye in standing position where gravity in positive $x$-direction (a) Velocity contour (b) Streamline and arrow surface (c) Pressure contour (d) Temperature contour

In order to confirm the dominance of temperature, effect of temperature difference on the $\mathrm{AH}$ flow is studied by simulating the eye in standing position model with no temperature difference as shown in Figure 5. The pattern of the $\mathrm{AH}$ flow is changed and there is no recirculation zone exist. $\mathrm{AH}$ enters through pupil aperture and leaves through TM at both sides without any recirculation. This indicated that the buoyancy effect is significant to drive the AH flow in AC. 


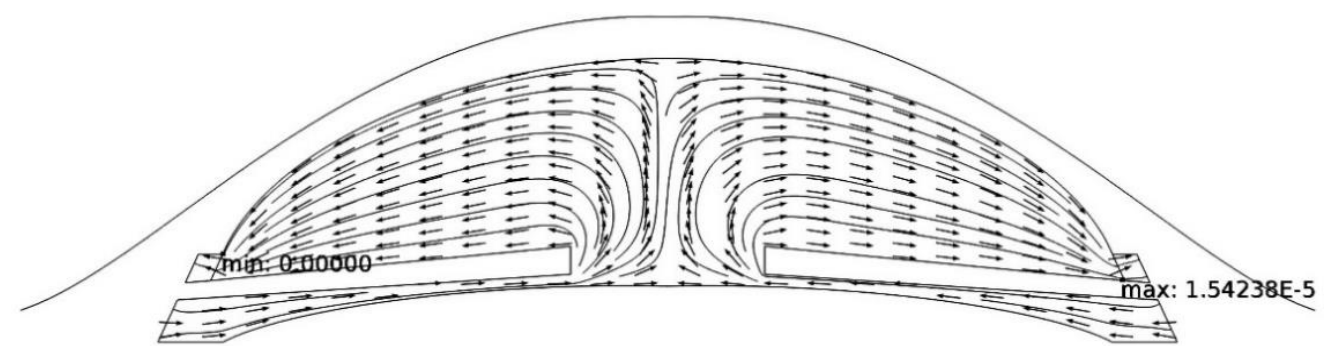

Fig. 5. AH flow for eye in standing position with no buoyancy effect or no temperature difference

Since the size of the pupil aperture varies according to light exposure in the surrounding, diameter sizes of $3 \mathrm{~mm}$ and $5 \mathrm{~mm}$ are simulated to observe the effect of pupil size. There are no any remarkable changes on the flow pattern of $\mathrm{AH}$. However, the maximum velocity increases when pupil sizes become larger. The results are display in Figure 6.

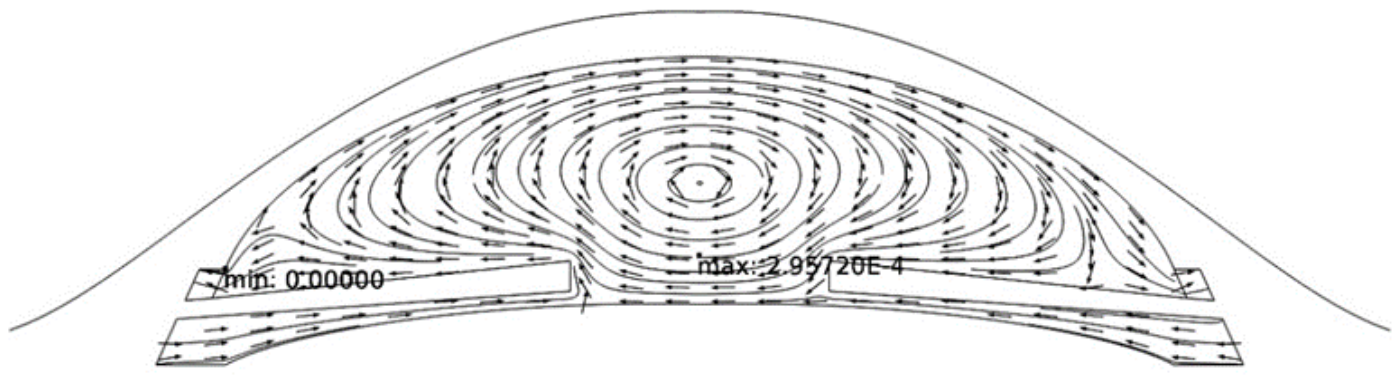

(a)

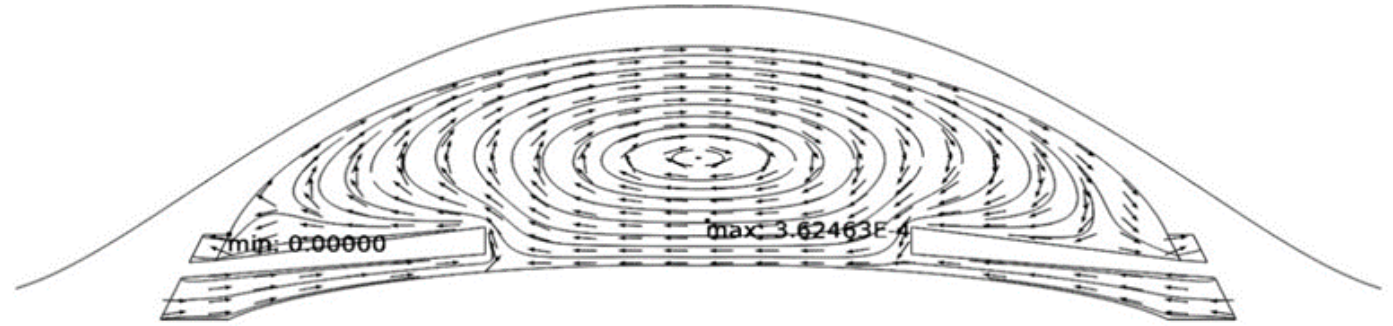

(b)

Fig. 6. Streamline plot of eye in standing position for different pupil sizes (a) Diameter of $3 \mathrm{~mm}$ (b) Diameter of $5 \mathrm{~mm}$

Next, for the model with the presence of DMD in standing position, the computational result of velocity are shown in Figure 7. The flow in middle part of $A C$ with presence of DMD is nearly the same as the model without DMD. Generally, the effect of TM resistance make most of the fluid not leave the $A C$ and move along the corneal surfaces. However, with the existence of DMD, the AH flows into the subspace between the DMD and cornea. The DMD moved away from the cornea as shown in Figure 7 because of the $\mathrm{AH}$ flow in the direction that pushed DMD farther from cornea.

On the other hand, the opposite direction of standing position or the negative $x$-direction of the gravitational forces is applied to investigate the $\mathrm{AH}$ flow in a more detailed way as presented in Figure 8. In this case, the movement of fluid in the subspace between the DMD and cornea is able to push the DMD closer to its original position. Reattachment could occur on the DMD if the gravity is in the negative $x$-direction. 


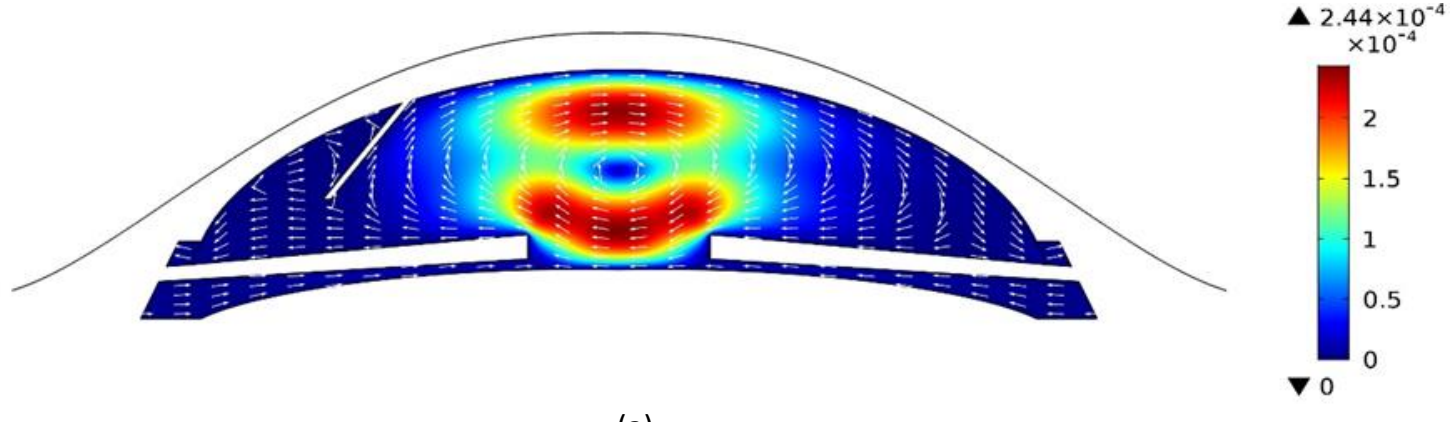

(a)

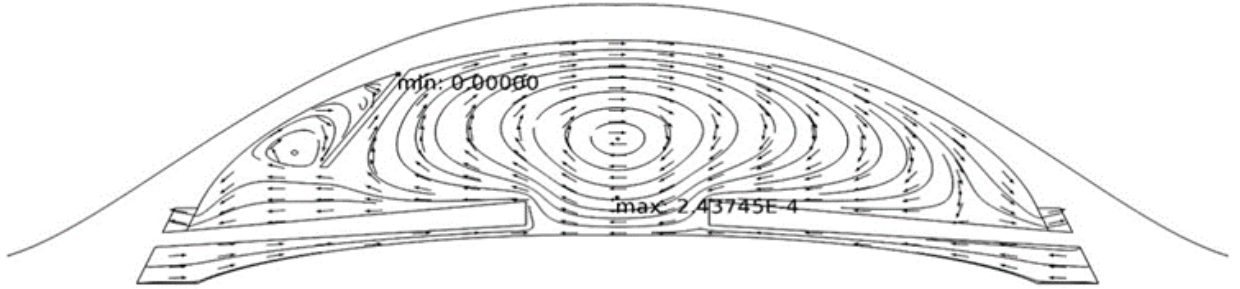

(b)

Fig. 7. AH flow for eye in standing position with DMD where gravity is in positive $x$-direction (a) Velocity contour (b) Streamline and arrow surface

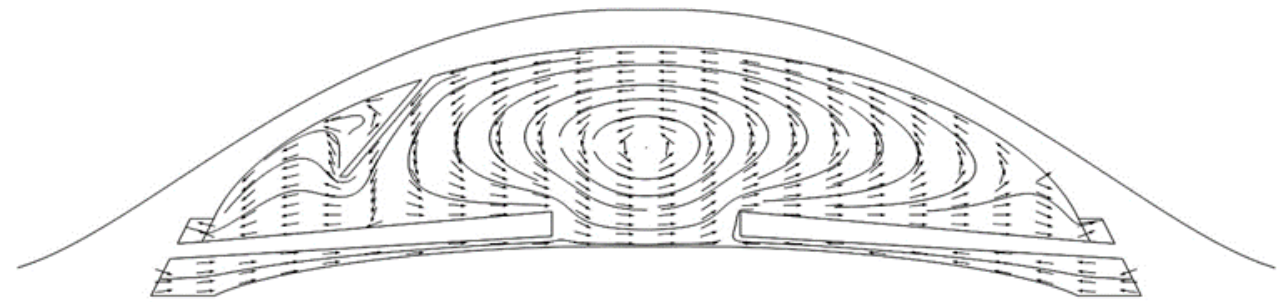

Fig. 8. Streamline plot for AH flow with DMD where gravity is acting in negative $x$ direction

\subsection{Eye in Supine Position}

In supine position, the body and gravitational forces are in the negative $y$-direction. Now examine the behaviour of $\mathrm{AH}$ flow in $\mathrm{AC}$ without $\mathrm{DMD}$ for supine position. The computational results are presented in Figure 9. The supine position creates a different circulating pattern of $A H$ in $A C$ compared to standing position. There are two large symmetric recirculating zones. The warmer fluid flows into pupil and rise upward towards the corneal surface in positive $y$-direction. The fluid descends downward along the corneal surfaces toward both sides of TM regions. This flow generates the two recirculating zones in AC. There is only a small portion of the fluid leaves the AC through the porous medium of TM.

The effect of buoyancy force is again studied in the present case. The eye in supine position with no temperature difference between the cornea and back of $A C$ is simulated and the streamline plot is displayed in Figure 10. The flow pattern is the same as the eye in standing position when no buoyancy effect and no recirculation occurred. Therefore, the temperature gradient is the driven mechanism of the $\mathrm{AH}$ flow. Hence, the buoyancy force of the temperature is responsible to the $\mathrm{AH}$ flow in AC.

The pupil size is fixed at the diameter of $2.4 \mathrm{~mm}$ throughout the study. Since in reality, the pupil size varies as discussed at previous section. The pupil size with diameter of $3 \mathrm{~mm}$ and $5 \mathrm{~mm}$ are modelled. The results showed that there are no significant changes on the $\mathrm{AH}$ flow pattern. The larger the diameter of pupil sizes, the larger the maximum velocity of AH flow in AC. The location of the 
maximum velocity in $\mathrm{AC}$ for diameter of $3 \mathrm{~mm}$ and $5 \mathrm{~mm}$ are displayed in the streamline plot as shown in Figure 11.
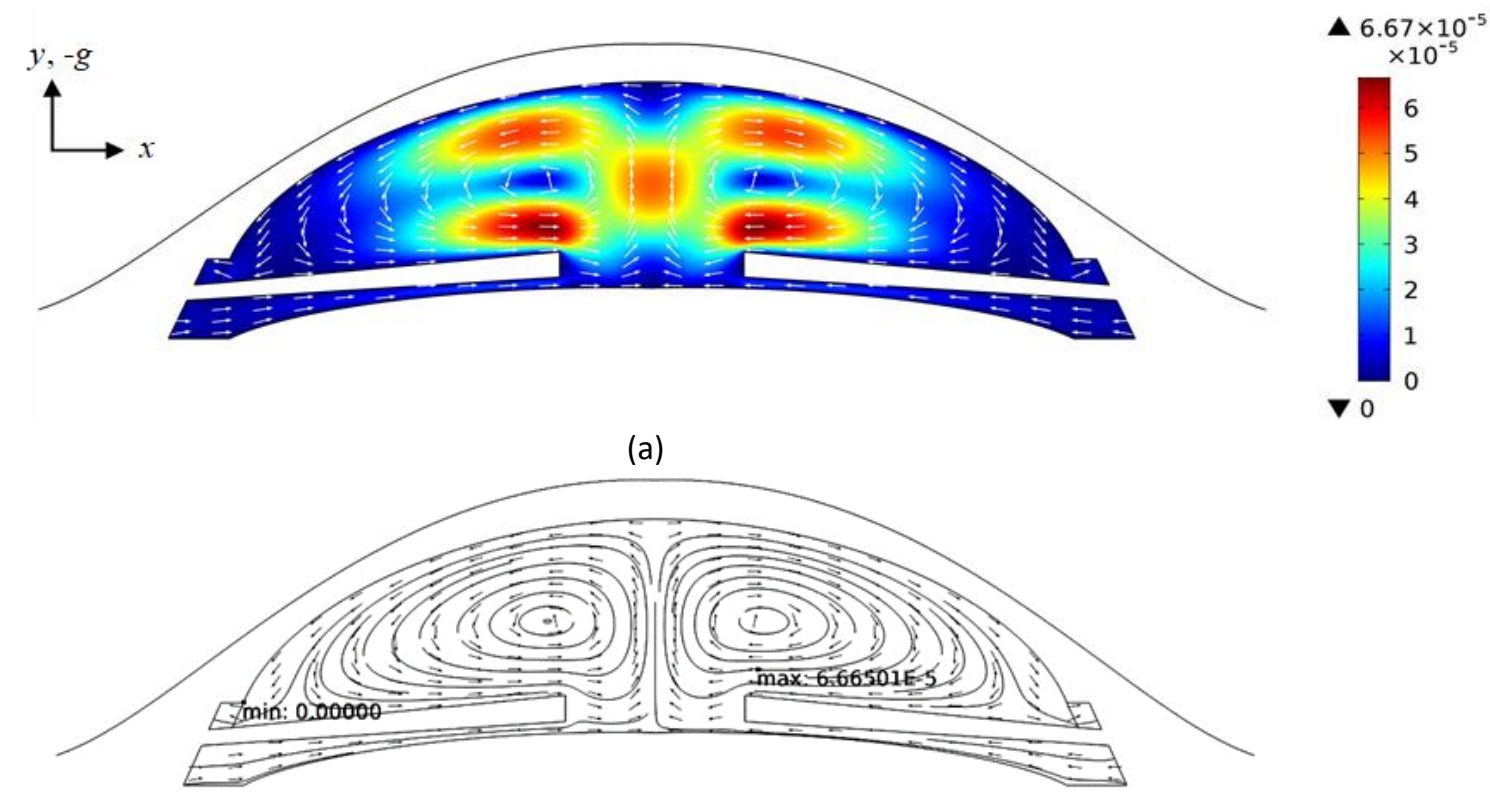

(b)

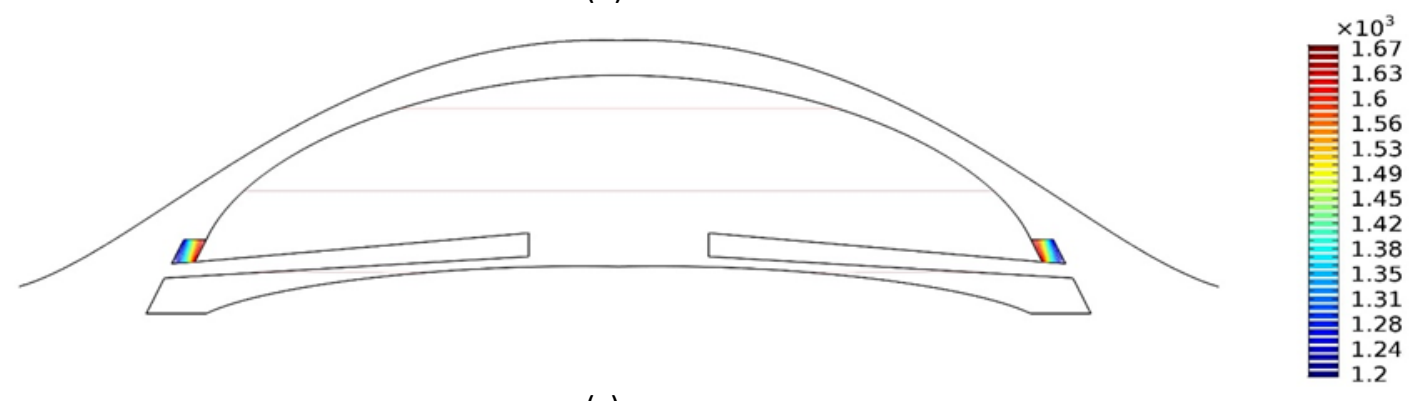

(c)

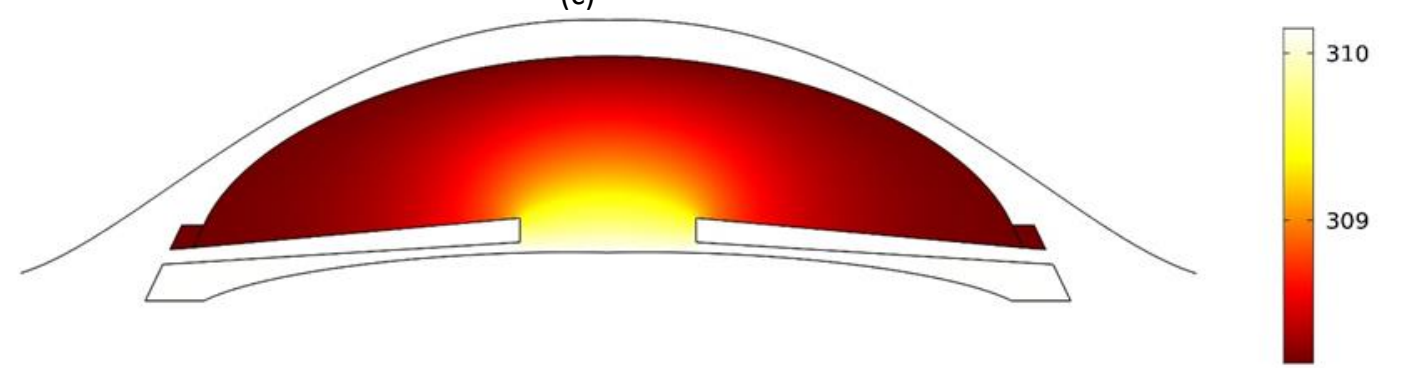

(d)

Fig. 9. AH flow for eye in supine position where gravity in negative $x$-direction (a) Velocity contour (b) Streamline and arrow surface (c) Pressure contour (d) Temperature contour

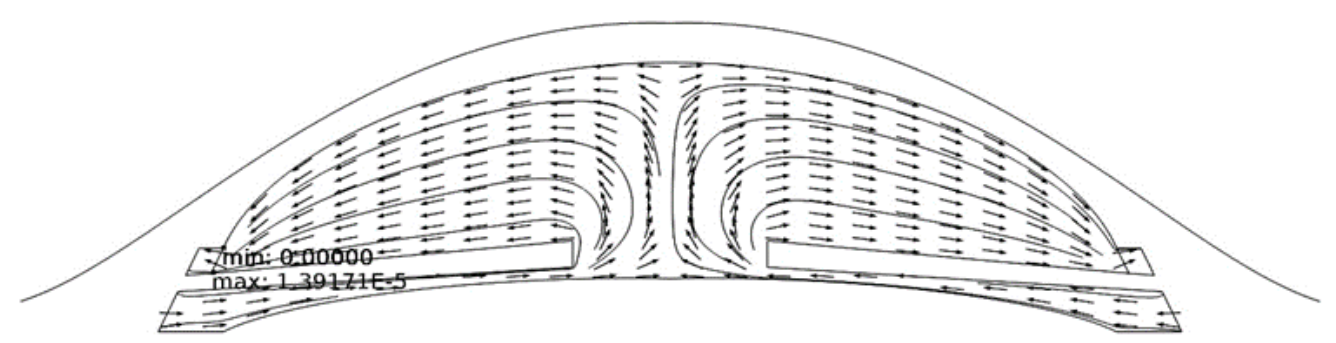

Fig. 10. AH flow for eye in supine position with no buoyancy effect or no temperature difference 


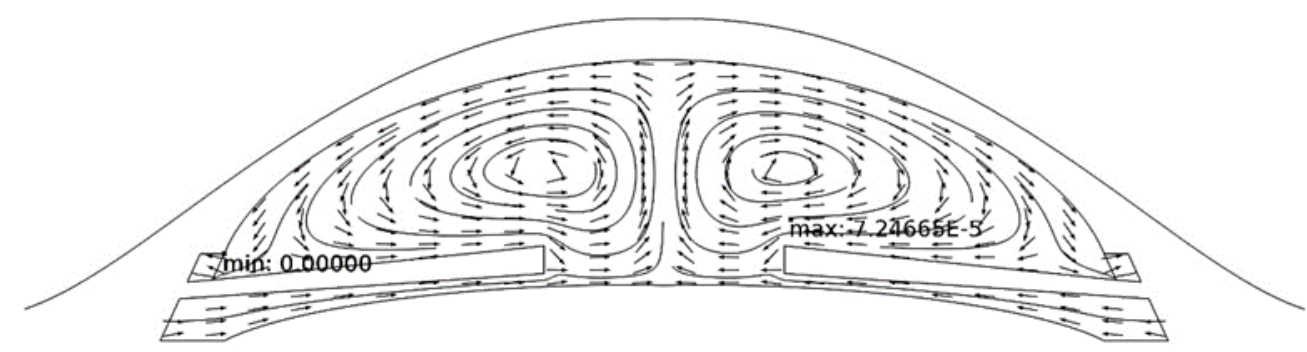

(a)

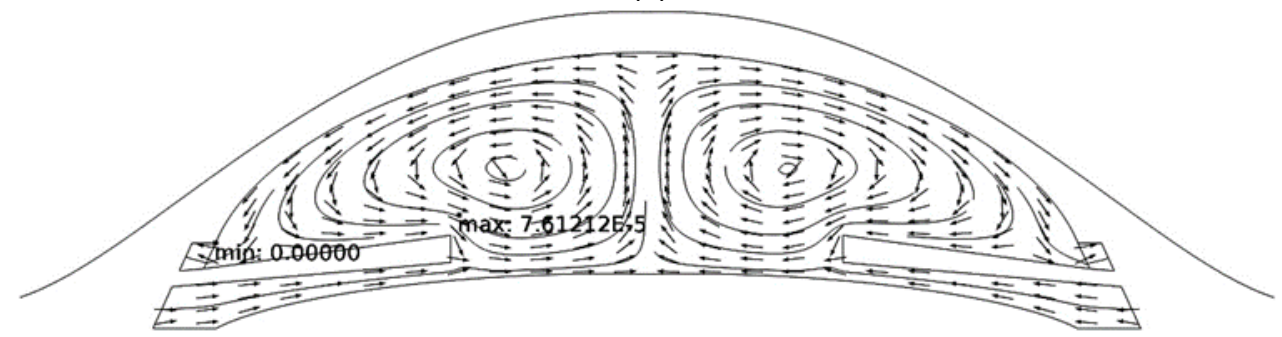

(b)

Fig. 11. Streamline plot of eye in supine position for different pupil size (a) Diameter of $3 \mathrm{~mm}$ (b) Diameter of $5 \mathrm{~mm}$

For the case of the model with DMD in supine position, the velocity contour and streamline plot are displayed clearly in Figure 12. The center region of $A C$ has the same circulating pattern as the model without DMD. However, the fluid flow moved upward to corneal surfaces and the fluid supposed to move downwards that encounters with the TM region. With the existence of DMD, AH flows into the region between DMD and cornea. The direction of the flow indicated that it is able to push DMD away from its original position.

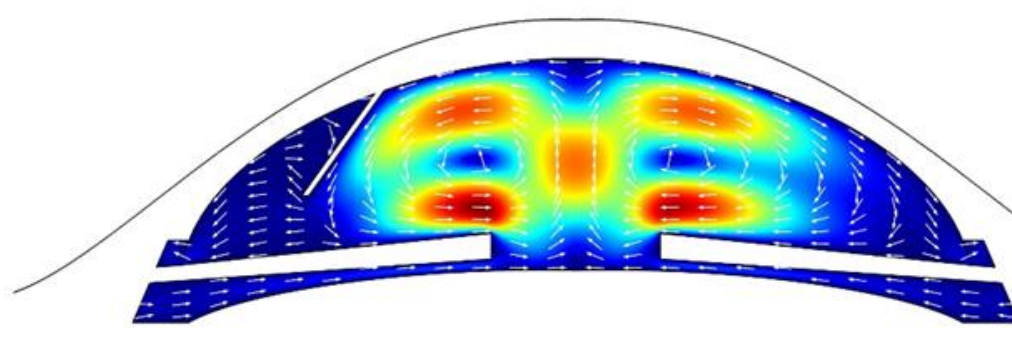

(a)

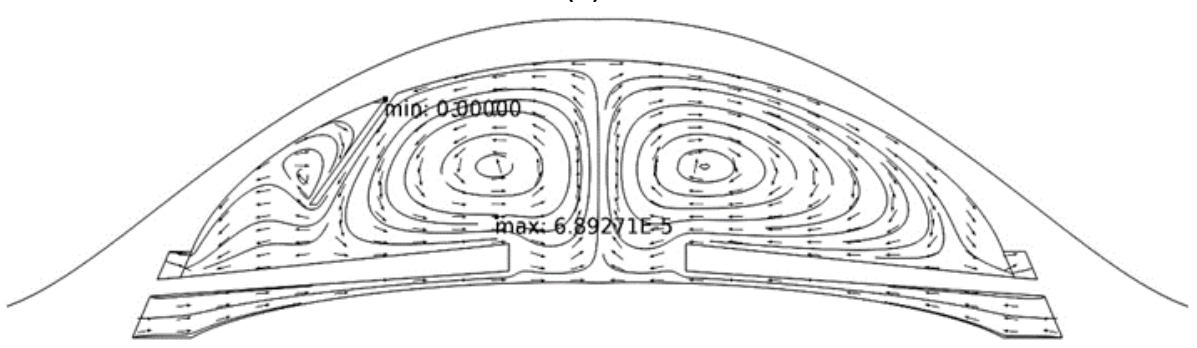

(b)

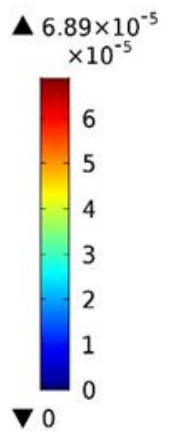


between the DMD and cornea, the AH flow in the direction towards cornea and DMD is moved in the direction towards its original position. The deformation of the DMD is closely related to the direction of the gravity acted since the gravity direction altered the $\mathrm{AH}$ flow in the AC.

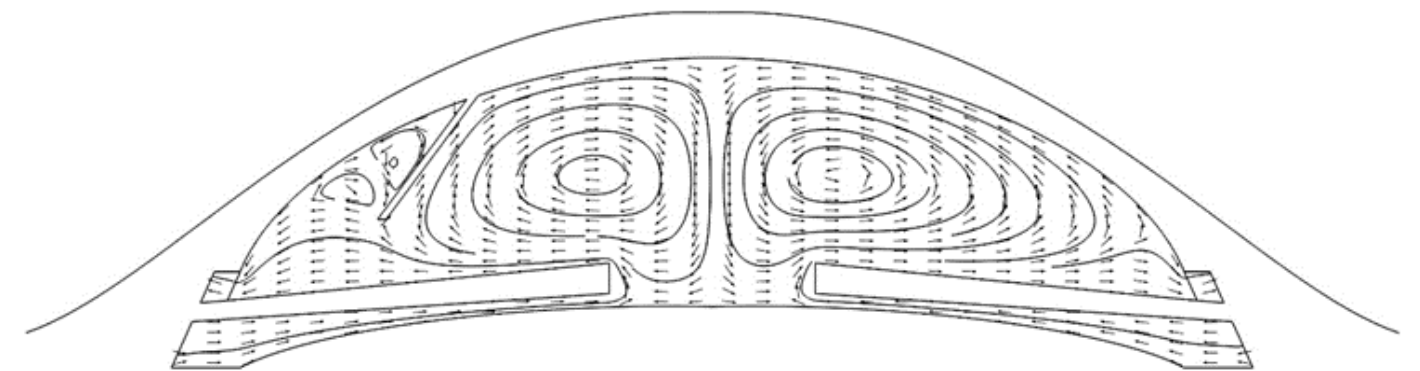

Fig. 13. Streamline plot for AH flow with DMD where gravity is acting in positive $y$-direction

From the results obtained in this research, the deformation of DMD is influenced by the gravity since the behaviour of fluid flow in $A C$ is different for the eye in supine and standing position. The reattachment of DMD can be done by adjusting the appropriate body position of the patient.

\section{Conclusions}

The two dimensional model of AC with the buoyancy driven AH enters through pupil aperture with DMD is studied numerically with the aiding of simulation software COMSOL Multiphysics 5.2. The eye in supine and standing position are taken into account to analyse the results. There are some interesting finding can be summarized as followed

i. The effect of pupil size is negligible because the different diameters of the pupil size do not change the pattern of the AH flow in the AC.

ii. The temperature difference between the cornea and iris are the driven mechanism of the $A H$ flow.

iii. The gravity has a significant effect on the AH flow. Hence, the effect of body position has to be considered in order to induce the reattachment of DMD.

iv. The method used in this study is suitable to simulate the flow of $A H$ in the $A C$ and it is applicable for considering different conditions.

It is suggesting to further investigate in three dimensional model so that a more accurate model can be simulated. More research can be done by considering the different types of DMD on the effect of $\mathrm{AH}$ flow through pupil aperture. Further research is believed to be necessary in order to fully investigate the behaviour of $\mathrm{AH}$ flow in the AC of human eye with the existence of DMD in order to improve and develop the treatment of DMD.

\section{Acknowledgement}

The authors would like to acknowledge Ministry of Higher Education and Research Management Centre, Universiti Teknologi Malaysia for the financial support through vote numbers Q.J130000.2554.21H48 (UTMFR Grant) and R.J130000.7854.5F255 (FRGS Grant). 


\section{References}

[1] Sevillano, C., E. Viso, and A. C. Millan-Rodriguez. "Descemet's membrane detachment as a complication of cataract surgery." Archivos de la Sociedad Espanola de Oftalmologia 83, no. 9 (2008): 549-551. https://doi.org/10.4321/s0365-66912008000900008

[2] Potter, Jessica, and Nadia Zalatimo. "Descemet's Membrane Detachment after Cataract Extraction." OptometryJournal of the American Optometric Association 76, no. 12 (2005): 720-24. https://doi.org/10.1016/j.optm.2005.08.028

[3] Ünlü, Kaan, and Ahmet Aksünger. "Descemet membrane detachment after viscocanalostomy." American journal of ophthalmology 130, no. 6 (2000): 833-834. https://doi.org/10.1016/s0002-9394(00)00628-0

[4] Couch, Steven M., and Keith H. Baratz. "Delayed, bilateral Descemet's membrane detachments with spontaneous resolution: implications for nonsurgical treatment." Cornea 28, no. 10 (2009): 1160-1163. https://doi.org/10.1097/ICO.0b013e318197eef1

[5] Ismail, Zuhaila. "The mathematical modelling of flow and deformation in the human eye." PhD diss., University of Southampton, 2013.

[6] Heys, Jeffrey J., and Victor H. Barocas. "A boussinesq model of natural convection in the human eye and the formation of Krukenberg's spindle." Annals of biomedical engineering 30, no. 3 (2002): 392-401. https://doi.org/10.1114/1.1477447

[7] Fitt, A. D., and G. Gonzalez. "Fluid mechanics of the human eye: aqueous humour flow in the anterior chamber." Bulletin of mathematical biology 68, no. 1 (2006): 53. https://doi.org/10.1007/s11538-005-9015-2

[8] Ismail, Z., A. D. Fitt, and C. P. Please. "A fluid mechanical explanation of the spontaneous reattachment of a previously detached Descemet membrane." Mathematical medicine and biology: a journal of the IMA 30, no. 4 (2013): 339-355.

https://doi.org/10.1093/imammb/dqs028

[9] Ooi, Ean-Hin, and Eddie Yin-Kwee Ng. "Simulation of aqueous humor hydrodynamics in human eye heat transfer." Computers in biology and medicine 38, no. 2 (2008): 252-262. https://doi.org/10.1016/j.compbiomed.2007.10.007

[10] Loke, Chai Y., Ean H. Ooi, Mohmed S. Salahudeen, Norlina Ramli, and Amir Samsudin. "Segmental aqueous humour outflow and eye orientation have strong influence on ocular drug delivery." Applied Mathematical Modelling 57 (2018): 474-491. https://doi.org/10.1016/j.apm.2018.01.007

[11] Jamali, Muhammad Sabaruddin Ahmad, and Zuhaila Ismail. "Simulation of Heat Transfer on Blood Flow through a Stenosed Bifurcated Artery." Journal of Advanced Research in Fluid Mechanics and Thermal Sciences 60, no. 2 (2019): 310-323.

[12] Ethier, C. Ross, Mark Johnson, and Jeff Ruberti. "Ocular biomechanics and biotransport." Annu. Rev. Biomed. Eng. 6 (2004): 249-273. https://doi.org/10.1146/annurev.bioeng.6.040803.140055

[13] Woan Jen, Woon. "Simulation of Aqueous Humour Flow Driven by Buoyancy Effect and Flow through Pupil Aperture During Descemet Membrane Detachment." Universiti Teknologi Malaysia, 2019.

[14] Canning, C. R., M. J. Greaney, J. N. Dewynne, and A. D. Fitt. "Fluid flow in the anterior chamber of a human eye." Mathematical Medicine and Biology: A Journal of the IMA 19, no. 1 (2002): 31-60. https://doi.org/10.1093/imammb/19.1.31

[15] Kumar, Satish, Sumanta Acharya, Roger Beuerman, and Arto Palkama. "Numerical solution of ocular fluid dynamics in a rabbit eye: parametric effects." Annals of biomedical engineering 34, no. 3 (2006): 530. https://doi.org/10.1007/s10439-005-9048-6 